\title{
Sistema de inferencia difuso para la valoración de empresas
}

\author{
Miguel David Rojas López* \\ Esteban Zuluaga Laserna* \\ Maria Elena Valencia Corrales***
}

Recibido: 11/09/2013 • Aceptado: 17/01/2014

\begin{abstract}
Resumen
El objetivo de esta investigación es proponer una herramienta basada en lógica difusa que permita implementarse en la valoración de empresas, admitiendo la inclusión de variables y criterios no cuantitativos en dicho proceso. Inicialmente, se exploran algunas de las principales metodologías de valoración de empresas, se identifican los factores que las impactan, y se establecen como insumo para la herramienta que propone esta investigación. Posteriormente, se presenta el marco teórico de lógica difusa y sobre el cual se sustenta la herramienta. Luego, se establecen las características en términos difusos de los componentes de la misma y se comparten algunos resultados. La herramienta presentada, además de incluir variables cualitativas y apoyar la toma de decisiones de inversión, permite el seguimiento a la gestión, ya que facilita medir las variaciones que algunas decisiones organizacionales generan en el valor de la empresa.
\end{abstract}

Palabras clave: valoración, lógica difusa, finanzas.

\footnotetext{
Ph. D. Ingeniería, Profesor Asociado,, Director Centro de Investigación y Consultoría Organizacional (CINCO), Departamento de Ingeniería de la Organización, Facultad de Minas, Universidad Nacional de Colombia, Sede Medellín. Carrera 80 N. 65-223 Bloque M8B oficina 202 Medellín, Tel 4255225-4255309. mdrojas@unal.edu.co

** Magíster en Ingeniería Administrativa, Investigador Centro de Investigación y Consultoría Organizacional Contabilidad Ambiente y Sociedad (CONTAS), Departamento de Ingeniería de la Organización, Facultad de Minas, Universidad Nacional de Colombia - Sede Medellín. ezuluag@unal.edu.co

*** Magíster en Administración, Investigador Centro de Investigación y Consultoría Organizacional (CINCO), Facultad de Ciencias Administrativas, económicas y contables, Fundación Universitaria Luis Amigó, Medellín. Maria.valenciaco@amigo.edu.co
} 


\title{
Diffuse inference system for valuation of companies
}

\begin{abstract}
The objective of this research is to propose a tool based on diffuse logic that would allow its implementation in the valuation of companies, accepting the inclusion of non-quantitative variables and criteria that have not been included in said process. Initially, some of the main company valuation methodologies are explored, identifying the factors that have an impact on them. Then, they are established as supply for the tool proposed in this research work. Subsequently, the diffuse logic theoretical framework is presented, which is the basis of the mentioned tool. Then, the characteristics are established in diffuse terms of its own components, and several results are disclosed. The presented tool, apart from including qualitative variables and supporting the investment decision-making process, allows the follow-up of the management as it facilitates the measurement of the variations that some organizational decisions generate in the value of the company.
\end{abstract}

Key words: valuation, diffuse logic, finance. 


\section{INTRODUCCIÓN}

La valoración de empresas es importante para las finanzas corporativas y la economía del negocio, dado que generalmente implica cambios en la estructura, estrategia y funcionamiento de la empresa.

La literatura financiera propone varios métodos para valorar empresas, donde predominan aquellos basados en el flujo descontado de fondos; los métodos presentan una estructura coherente pero que excluye variables concernientes a la estrategia, calidad de los productos, habilidad de las directivas, conocimiento del mercado y el análisis del sector al cual pertenece la empresa. Otros métodos, como el análisis de indicadores o estados financieros, no son ajenos a este problema. La valoración es una caja negra; no se pueden estimar las cifras en el proceso subjetivo y cognoscitivo, ni intentar pronosticar los supuestos con los cuales se desarrolló el mismo, es decir, no se logra un proceso articulado y replicable.

La administración estratégica se preocupa más por los análisis cualitativos, argumentando que la empresa puede generar valor desde la estructura del sector al cual pertenece, competencias internas y gestión de los recursos propios, valorando la empresa por su presente y futuro en el entorno.

Esta investigación propone una herramienta basada en lógica difusa, que incluya esas variables y criterios cualitativos y apoye el proceso de valoración. Para esto, es necesario conocer algunas metodologías de valoración y los conceptos de lógica difusa, estableciendo las variables involucradas y la herramienta.

\section{METODOLOGÍAS PARA LA VALORACIÓN DE EMPRESAS}

Las empresas son valoradas por tres motivos (ver tabla 1).

Tabla 1: Motivos de la valoración.

\begin{tabular}{|l|l|}
\hline $\begin{array}{l}\text { Conflictos } \\
\text { legales }\end{array}$ & $\begin{array}{l}\text { Participación en herencias, procesos de expropiación, divorcios, liquidación en caso } \\
\text { de quiebra. }\end{array}$ \\
\hline $\begin{array}{l}\text { Oportunidades } \\
\text { de mercado }\end{array}$ & $\begin{array}{l}\text { Operaciones de compra y venta de empresas, emisión de títulos, definición de portafolios, } \\
\text { privatizaciones, fusiones, integraciones o capitalizaciones. }\end{array}$ \\
\hline $\begin{array}{l}\text { Cambios } \\
\text { estructurales } \\
\text { internos }\end{array}$ & $\begin{array}{l}\text { Sistemas de remuneración, planeación estratégica, identificación de eslabones genera- } \\
\text { dores de valor, políticas de dividendos (Caballery Ballestero, 1998; Fernández, 2004), } \\
\text { y su objetivo final es obtener información para la toma de decisiones permitiendo apro- } \\
\text { vechar las oportunidades de alcanzar beneficios. Esto implica, entonces, que existe una } \\
\text { transferencia de valor que requiere su valoración (Santandreu, 1990). }\end{array}$ \\
\hline
\end{tabular}

Fuente: elaboración propia

Las metodologías para la valoración de empresas se clasifican en cinco grupos: basadas en la contabilidad (balance), basadas en indicadores, las mixtas (o Goodwill), las de descuentos de flujos futuros, y las de valor creado [1]. 


\subsection{Basadas en el balance}

El valor de una compañía se encuentra en el balance, y se determina dicho valor mediante la estimación del patrimonio. Algunos de los métodos de esta categoría son: valor contable (patrimonio neto), valor contable ajustado, valor de liquidación y valor sustancial. El inconveniente de estos métodos es que obtienen el valor de la empresa desde una perspectiva estática, que no considera factores ajenos a la elaboración de los registros contables, como: futuro de la compañía, valor del dinero en el tiempo, situación del sector y problemas humanos [2]. La valoración estática sirve como base de comparación contra otros métodos y como método auxiliar [3].

\subsection{Basadas en indicadores}

Son estáticos, evaluando las obligaciones, derechos y bienes de una empresa. El método obtiene un índice que compara diferentes cuentas de la empresa como patrimonio, ventas, utilidades u otra, y que, a su vez, es comparable con el de otra empresa, analizado para obtener conclusiones específicas. Los métodos basados en indicadores son divididos en tres grupos: patrimoniales, estratégicos y de rendimiento [2].

\subsubsection{Patrimoniales}

Se valora el patrimonio sin considerar la capacidad para generar excedentes en el futuro. Este método contempla principalmente tres formas de aplicación:

El valor de la empresa (VE) comparado con el patrimonio como el valor contable del mismo (VCP). El índice patrimonial es:

$$
I P_{1}=\frac{V E}{V C P}
$$

El valor de la empresa comparado con el patrimonio como el valor de reposición a precios de mercado de los activos (VPM), descontando la deuda (D). El índice patrimonial para esta forma es:

$$
I P_{2}=\frac{V E}{V C P-D}
$$

El valor de la empresa comparado con el patrimonio como el valor patrimonial neto $(\mathrm{VPaN})$, el cual considera los ajustes necesarios para obtener una valoración a precios de mercado, como los pasivos contingentes y las plusvalías latentes. El índice patrimonial para esta forma es:

$$
I P_{3}=\frac{V E}{V P a N}
$$


Para el comprador estos indicadores deben ser menores a 1.

\subsubsection{Estratégicos}

Con los procesos de internacionalización vividos por las economías durante el período comprendido entre 2000 y 2005 por medio de los tratados de libre comercio, se han observados en Latinoamérica amplios procesos de adquisición y fusión de empresas, con el propósito de ampliar su participación y lograr de esta manera hacer frente a los competidores que llegarían de otros países; son los casos de Zenú, Noel y algunas cementeras [4].

Los indicadores estratégicos captan los motivos de los factores económicos o financieros mediante multiplicadores basados en la experiencia del mercado.

Los pasos para la aplicación del método son:

a. Elaborar un indicador que compare el valor o precio de la empresa con el atributo estratégico deseado.

b. Establecer desde la experiencia un nivel estándar para el indicador.

c. Aplicar el indicador con su valor estándar al atributo estratégico.

La metodología tiene dos debilidades: una, carece de rigor financiero al no incluir el rendimiento de las inversiones; y dos, supone homogeneidad en las empresas.

\subsubsection{De rendimiento}

Origen del método de descuento de flujo de caja. Se emplean tres indicadores:

$$
P E R=\frac{P E}{B} P C F R=\frac{P E}{F C T} D . Y=\frac{D T}{P Y}
$$

$\mathrm{PE}=$ Precio de la empresa

$\mathrm{PER}=$ Price earnings ratio. . Indicador ganancia precio.

$\mathrm{B}=$ beneficio o utilidades.

PCFR $=$ Price cash flow ratio. Indicador del flujo de capital neto.

$\mathrm{FCT}=$ Flujo de capital total.

D.Y = Dividen yield . Renta por dividendo.

El indicador más empleado es el PER, que considera la capacidad actual para generar beneficio de la empresa a comprar o vender.

Para cualquiera de los indicadores es importante analizar si está sub o sobre valorado. Se recomienda el uso de beneficios periódicos y no ocasionales. Los resultados 
obtenidos por esta metodología son menos ajustados que los obtenidos por medio del descuento de flujos. Es recomendado como apoyo.

\subsection{Mixtos}

Buscan conjugar las valoraciones contables y el análisis financiero. Plantean la descomposición del valor de la empresa en el monto inicial de las inversiones y la cuantía derivada de su capacidad de generar retornos superiores a los exigibles en función del riesgo. Son sencillos y requieren poca información.

$\mathrm{Su}$ mayor aporte frente a los contables y a los basados en indicadores es que añaden al valor sustancial (de los activos) la capacidad de generación de los recursos (excedentes) que tiene la empresa [1]. (ver tabla 2)

Tabla 2. Métodos mixtos de valoración de empresas.

\begin{tabular}{|l|c|}
\hline \multicolumn{1}{|c|}{ Método } & Forma de cálculo \\
\hline Clásico & $V E=A+\left(n \times B^{0}\right)$ \\
\hline Renta abreviada del Goodwill & $V E=A+a_{n} \times\left(B^{0}-R_{f} \times A\right)$ \\
\hline Unión de expertos contables & $V E=\frac{A+a_{n} \times B^{0}}{\left(1+a_{n} \times R_{f}\right)}$ \\
\hline Prácticos o indirectos & $V E=A+\frac{\left(B^{0}-R_{f} \times A\right)}{2 \times R_{f}}$ \\
\hline Anglosajón o directo & $V E=A+\frac{\left(B^{0}-R_{f} \times A\right)}{k}$ \\
\hline Compra de resultados anuales & $V E=A+m \times\left(B^{0}-R_{f} \times A\right)$ \\
\hline Tasa con riesgo y libre de riesgo & $V E=\frac{A+\frac{B^{0}}{k}}{1+\frac{R_{f}}{k}}$ \\
\hline
\end{tabular}

Fuente: elaboración fuente propia con base en Fernández (2004), Viñolas y Adserá (2003), Caballer (1998) y Santandreu (1990).

$\mathrm{VE}=$ Valor de la empresa

$\mathrm{A}=$ Valor de los activos netos ajustados a precios de mercado 
$\mathrm{n}=$ Múltiplo del beneficio.

$\mathrm{B}^{0}=$ Beneficios generados por la empresa

$\mathrm{a}_{\mathrm{n}}=$ Factor de actualización de $\mathrm{n}$ unidades

$\mathrm{R}_{\mathrm{f}}=$ Tasa de interés libre de riesgo

$\mathrm{k}=$ Tasa de interés con riesgo

$\mathrm{m}=$ Número de años de superbeneficio que se valora el goodwill.

Los métodos mixtos son criticados por considerarse teóricamente incorrectos; las principales debilidades son:

- Los activos no siempre reflejan su verdadero valor, y se asume que la empresa está en capacidad de generar valor por encima de sus activos.

- No se consideran las inversiones necesarias para generar beneficios ni el crecimiento esperado de los flujos futuros.

- La valoración es estática.

- Los múltiplos no tienen una forma de cálculo basada en comparaciones con otros utilizados en valoraciones similares.

\subsection{Descuento del flujo de fondos}

Considerado el método técnico más apropiado para la valoración de empresas. Sus bases son los fondos esperados y una tasa exigida acorde con el riesgo de esperar dichos fondos [5]. Estos métodos reconocen que para crear valor se requiere de inversiones, y que una empresa que necesite menos capital por unidad de beneficio debe ser premiada con una mejor valoración [3].

Las principales ventajas según [1] son:

- $\quad$ Su base son los flujos de caja y no magnitudes contables.

- Recopila información del balance y del estado de resultados.

- Refleja situaciones coyunturales.

Considera el valor del dinero en el tiempo.

El valor de la empresa se estima como la valoración a corto plazo (VC), que incorpora situaciones coyunturales; y a largo plazo (VL), o estructural.

$$
V E=V C+V L ; V C=\sum_{j=1}^{n} \frac{C F_{j}}{(1+k)^{j}} ; V L=\frac{C F_{n} \times(1+g)}{k-g}
$$

$\mathrm{CF}=$ Valor de los flujos de caja en el corto plazo 
$\mathrm{k}=$ Tasa de descuento

$\mathrm{g}=$ Tasa de crecimiento a perpetuidad (fija) de la empresa.

Esto implica determinar la tasa de crecimiento a la que el flujo se incrementará en el futuro y la tasa de descuento que se aplicará a los flujos venideros.

La determinación de la tasa de descuento de flujos es un aspecto a tener en cuenta, así como el riesgo y las volatilidades históricas. Representa el costo de oportunidad de los recursos invertidos en la empresa y el grado de riesgo asociado a la inversión.

\subsection{Basadas en la creación de valor}

El objetivo de la dirección de la empresa es maximizar su valor. Los principales generadores de valor se pueden clasificar en operativos y financieros; los operativos se derivan de la estrategia competitiva de la empresa; los financieros se relacionan con el rendimiento de la inversión, el nivel de endeudamiento o el costo de los recursos. Generar valor implica gestionar los conductores clave para la empresa, y esta gestión determina la capacidad de generar fondos en el futuro.

Algunos de los métodos más utilizados son: valor económico agregado (EVA®), beneficio económico, valor de mercado añadido (MVA), valor de caja adicionado (CVA), rentabilidad del flujo de la inversión (CFROI).

No es lo mismo la rentabilidad económica que la rentabilidad contable, dado que la última no contempla factores externos que pueden afectar los resultados futuros (Medina, 2002). Para medir la generación de valor y el valor de una empresa, se deben tener en cuenta tres elementos determinantes: rentabilidad esperada, riesgo inherente y tiempo que puede mantener dicha rentabilidad.

\subsection{Sistemas de lógica difusa}

La búsqueda de soluciones a problemas que parten de las percepciones recurren a modelos alternativos que reflejen de alguna manera numérica variables de carácter lingüístico. La lógica difusa-LF se presenta como una herramienta para lograr esta transformación; sus fundamentos son los conjuntos difusos según los cuales el grado de pertenencia de un elemento a un conjunto está determinado por una función de pertenencia que puede tomar todos los valores reales comprendidos en el intervalo $[0,1][6-9]$. LF permite definir grados variables de pertenencia que se asemejan, en razonamiento y patrones, al pensamiento humano, dejando conceptos determinísticos tradicionales.

Actualmente, cualquier relación entre variables de entrada será aproximada por medio de un sistema difuso construido en términos lingüísticos con alto grado de exactitud [9-10]. Los pasos que integran un sistema de inferencia difuso son: 
Parametrización: se definen las variables de entrada y salida.

Reglas difusas si-entonces: relacionan las variables de entrada y salida, determinando el grado de pertenencia. Se utilizan las relaciones tipo Mandami, que plantean que: "Si $\mathrm{X}_{1}$ es $\mathrm{A}_{1} \mathrm{y}_{2}$ es $\mathrm{A}_{2} \mathrm{y} \mathrm{X}_{3}$ es $\mathrm{A}_{3}$ entonces $\mathrm{Y}$ es B".

Operaciones de composición. Las encargadas de concretar el resultado difuso por medio de un operador [11-12, 6-8].

Mecanismos de inferencia: permite inferir y concluir.

Agregación: las variables resultantes de las reglas son agregadas.

Proceso de concreción. Parte del conjunto difuso de salida para obtener un valor concreto que proporciona la solución del sistema que se planteó.

\section{HERRAMIENTA PARA LA TOMA DE DECISIONES DE INVERSIÓN}

El valor de una empresa se calcula con criterios que varían entre analistas. Tres conceptos se hacen evidentes: el precio, como resultado del intercambio de unidades monetarias; el costo, que son los recursos necesarios para mantener la empresa, y el valor, que refleja la capacidad para generar beneficios a sus accionistas o beneficiarios.

Cuanto más complejo el bien, más difícil resulta determinar su coste, sobre todo cuando su elaboración se prolonga a lo largo de un período de tiempo; lo anterior, dando lugar a agregar costes que no son homogéneos [2]. Desde la teoría microeconómica de la preferencia del consumidor se plantea que un mismo bien consumido o adquirido en dos lugares o circunstancias distintas equivale a dos bienes diferentes, ya que el comprador puede valorarlo de forma diferente [13].

Una valoración correcta es aquella que está bien fundamentada técnicamente y se basa en supuestos razonables o correctos, teniendo en cuenta la perspectiva subjetiva bajo la que se lleva a cabo la valoración [14]. Esta subjetividad presenta el problema de agregación y comparación; los modelos presentados en la sección 1 no logran por sí solos una valoración unificada, y son insuficientes como componentes independientes.

En esta investigación se unen diferentes componentes de los modelos presentados en la sección 1, que influyen en la percepción del valor de la empresa.

Para la implementación de esta herramienta se utilizarán, por fines prácticos, conjuntos triangulares para las variables de entrada y salida, como se muestra en la figura 1. 


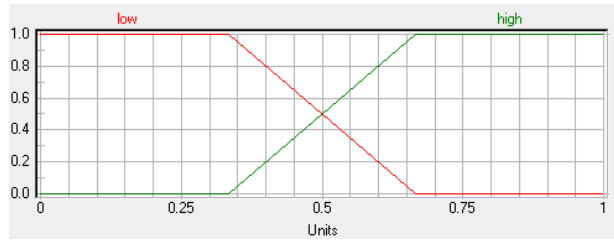

a) Variables de entrada

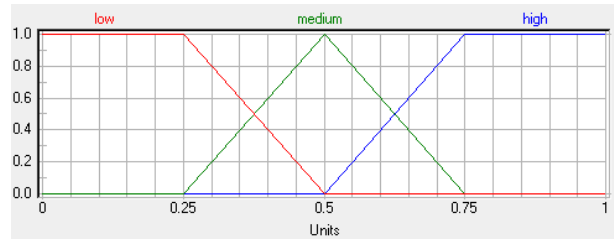

b) Variables de salida

Figura 1. Conjuntos difusos triangulares para las variables de entrada y salida.

Fuente: elaboración propia

\subsection{Competencias internas}

Corresponden a competencias directamente ligadas a la gestión de la empresa en el mercado y con los clientes. Son resultado del goodwill, buenos indicadores de generación.

El sistema de inferencia difuso para esta variable se presenta en la figura 2.

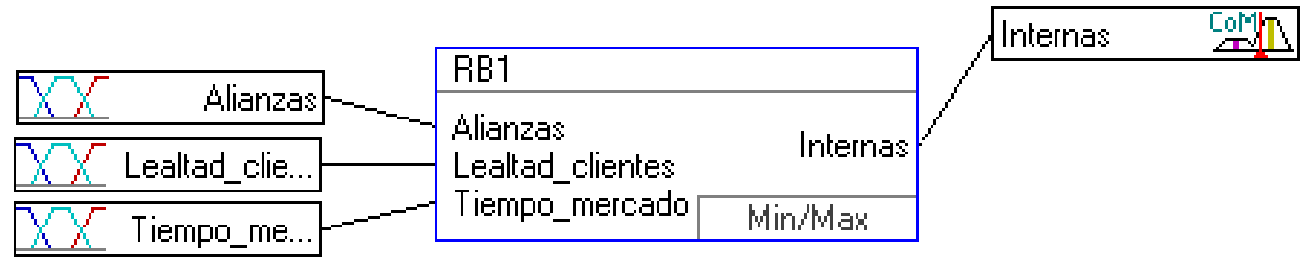

Figura 2. Estructura del sistema de inferencia para las competencias internas Fuente: elaboración propia

La variable competencias internas considera dos conjuntos difusos definidos:

Altas competencias internas: Referencia a competitividad operacional y estratégica. Bajas competencias internas: se presenta cuando la operación de la empresa no es rentable, los clientes no son leales y se tienen períodos intermitentes de trabajo. Las variables de entrada para las competencias internas se definen como:

\subsubsection{Alianzas estratégicas}

Su importancia radica en que hay un alto desarrollo de tecnologías de comunicación que apoyan las alianzas; además, permiten entrar a los mercados específicos con mayor rapidez, reducen riesgos financieros, políticos, competencia, mejoran accesos a recursos, entre otras. [15].

Los conjuntos difusos que se consideran para medir las alianzas estratégicas son: Alianzas estratégicas altas, un tipo de alianza óptima, con estrategia definida.

Alianzas estratégicas medias, no aprovechan al máximo el potencial de la alianza.

Alianza estratégicas bajas, cuyos resultados no son los esperados. 


\subsubsection{Tiempo en el mercado}

Es el cociente entre el tiempo de mercado promedio de una empresa y el tiempo de mercado promedio en el sector [16].

Los conjuntos difusos que se consideran para medir el tiempo en el mercado son:

Mucho tiempo en el mercado, toman decisiones con mayor rapidez.

Normal tiempo en el mercado, posiblemente innovaron en el mercado.

Poco tiempo en el mercado, no logran posicionamiento en el sector.

\subsubsection{Lealtad de los clientes}

Se relaciona con la preferencia por los productos y los servicios de la empresa, con una recurrencia o continuidad en la adquisición de estos. Los conjuntos difusos que se consideran para medir la lealtad de los clientes son:

Alta lealtad de los clientes, dan estabilidad a la empresa.

Media lealtad de los clientes, existe el riesgo de perder a los clientes.

Baja lealtad de los clientes, los clientes tienden a cambiar de proveedor.

La base de conocimiento se construyó con apoyo de un panel de expertos, que analizó las relaciones implícitas entre las variables de entrada y salida del sistema definido. Estas relaciones son representadas en forma de reglas del tipo si-entonces.

Una de dichas relaciones puede ser expresada de la siguiente forma: si la lealtad de los clientes es baja y está asociada a bajas alianzas y a un corto tiempo en el mercado, las competencias internas serán bajas. Esto significa que la empresa no está aprovechando sus competencias o que la dirección no está tomando decisiones encaminadas a la generación de valor.

De la base de conocimiento para la medición de las competencias internas se presenta, en la tabla 3 , una parte, porque deberían mostrarse todas las combinaciones posibles.

Tabla 3. Base de conocimientos para la variable competencias internas.

\begin{tabular}{|c|c|c|c|}
\hline \multicolumn{3}{|c|}{ IF } & THEN \\
\hline Alianzas & Lealtad_clientes & Tiempo_mercado & Internas \\
\hline Bajas & Baja & Poco & Bajas \\
\hline Bajas & Baja & Normal & Bajas \\
\hline Bajas & Baja & Mucho & Bajas \\
\hline
\end{tabular}

Fuente: elaboración propia 


\subsection{Competencias monetarias}

Son las competencias propias de la empresa, es decir, que pueden ser influenciadas y establecidas por las decisiones de las directivas. Impactan la generación futura de beneficios desde la operatividad y la estrategia. Son propias del flujo de caja, los indicadores estratégicos y la percepción del analista de futuras oportunidades.

El sistema de inferencia difuso para esta variable se presenta en la figura 3.

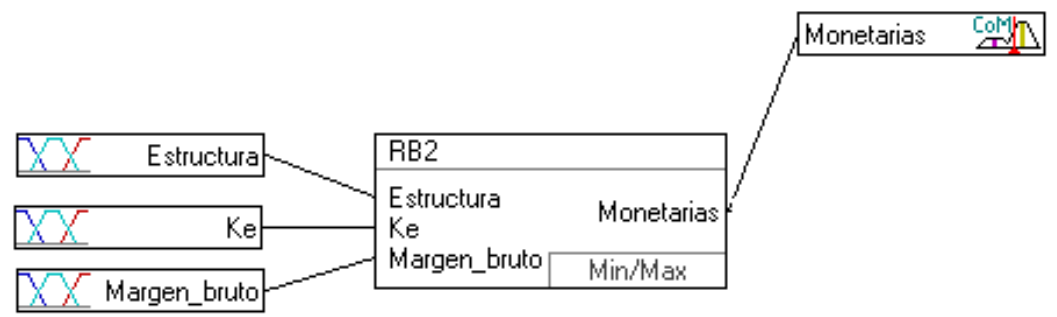

Figura 3. Estructura del sistema de inferencia para las competencias monetarias

Fuente: elaboración propia

Se consideran dos conjuntos difusos definidos como sigue:

Altas competencias monetarias, empresas que han logrado un equilibrio en su estructura financiera.

Bajas competencias monetarias, dirección financiera y operativa con falencias considerables.

Las variables de entrada para las competencias monetarias se definen como:

\subsubsection{Margen bruto}

El margen bruto se define como los ingresos, menos lo que cuesta la fabricación del producto [17]. Exponen comparativamente la diferencia existente entre el valor bruto de la producción y el costo directo asociado a dicha actividad.

El criterio basado en el ingreso establece que el valor de un activo es el valor presente de sus retornos esperados; se espera que un activo provea retornos durante el período que se posea; para convertir esta serie de retornos en un valor confiable, se debe descontar a la tasa de descuento [18].

Los conjuntos difusos que se consideran para medir el margen bruto son:

Margen bruto alto, se presenta una buena eficiencia en las operaciones y una adecuada asignación de los precios a los productos.

Margen bruto medio, la empresa no utiliza al máximo los recursos de producción.

Margen bruto bajo, el costo de la mercancía supera los ingresos por las ventas. 


\subsubsection{Estructura financiera}

Es el total de los pasivos dividido el total del patrimonio [17]. Es financiar los activos incluyendo el capital de los accionistas y deuda, a corto y largo plazo.

El financiamiento se logra utilizando los recursos propios de la empresa o a través de deuda contraída por medio de un sistema financiero. El modelo de apalancamiento objetivo (trade off) sostiene que las empresas buscan un apalancamiento óptimo, es decir, aquel que minimice el costo de capital [19].

Los conjuntos difusos que se consideran para medir la estructura financiera son:

Estructura financiera alta, organizaciones que utilizan al mercado de capitales como su principal opción de financiamiento.

Estructura financiera media, fondo a corto y largo plazo.

Estructura financiera baja, mezcla de fuentes de fondo de corto plazo e inversión.

\subsubsection{Costo del patrimonio}

El costo del Equity es la tasa de retorno que la empresa paga a sus accionistas por la obtención del capital que ellos proveen. El costo de capital de la empresa se definiría entonces como un promedio ponderado del capital invertido de cada una de las fuentes de capital a la tasa que cada uno de ellos está invirtiendo en la empresa; si una empresa está creando o destruyendo valor, este promedio es un elemento básico, junto con el retorno sobre el capital invertido (ROI), ya que siempre que el ROI supere el costo del capital invertido, crea valor [20].

Los conjuntos difusos que se consideran para medir el costo de capital son:

Costo de Equity alto, inversión del accionista supera sus expectativas.

Costo de Equity medio, la tasa de retorno es la esperada por el inversionista.

Costo de Equity bajo, no se utiliza correctamente el potencial de la empresa.

La base de conocimiento para la medición las competencias monetarias se debe presentar con todas las combinaciones posibles; se presenta una parte en la tabla 4.

Tabla 4. Base de conocimientos para la variable competencias monetarias.

\begin{tabular}{|c|c|c|c|}
\hline \multicolumn{3}{|c|}{ IF } & THEN \\
\hline Estructura & Ke & Margen_bruto & Monetarias \\
\hline Baja & Bajo & Bajo & Bajas \\
\hline Baja & Bajo & Medio & Altas \\
\hline Baja & Bajo & Alto & Altas \\
\hline
\end{tabular}

Fuente: elaboración propia 


\subsection{Aspectos macroeconómicos}

Corresponden al efecto que la economía podría tener sobre el rendimiento de la empresa. Comprometen la percepción de los flujos futuros y limitan algunas de las proyecciones con el fin de ajustar los planteamientos a la realidad. Afectan fuertemente la percepción del analista sobre la posibilidad de cumplir los escenarios planteados y obtener rendimientos proyectados.

El sistema de inferencia difuso para esta variable se presenta en la figura 4.

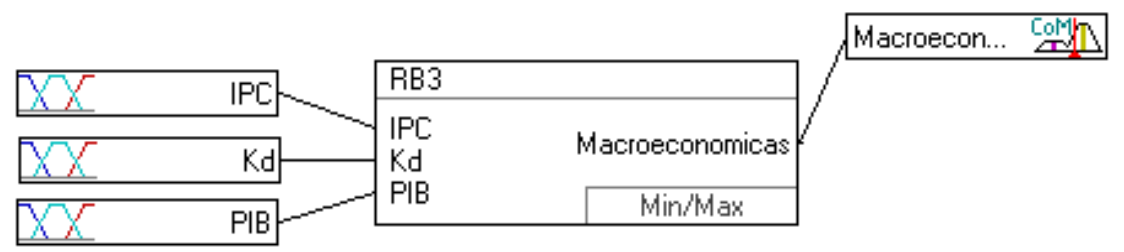

Figura 4. Estructura del sistema de inferencia para los aspectos macroeconómicos.

Fuente: elaboración propia

Para la variable aspectos macroeconómicos, se consideran dos conjuntos difusos definidos como sigue:

Aspectos macroeconómicos favorables, se presentan señales que permiten inferir un comportamiento macroeconómico propicio para la empresa, en el sector y el país.

Aspectos macroeconómicos desfavorables, incrementan la percepción del entorno macroeconómico contraproducente para la empresa. Las variables de entrada para las competencias monetarias se definen como:

\subsubsection{PIB}

La actividad económica de cualquier país se mide a través de sus cuentas nacionales. Es la información referente a aspectos macroeconómicos como: producción, consumo, ahorro, inversión, transacciones financieras y las relaciones económicas con el exterior. Entre los economistas, el enfoque del gasto es el más utilizado (Álvarez y Sebastián, 1998). Generalmente se le denomina ecuación keynesiana y se expresa como: PIB = $\mathrm{C}+\mathrm{I}+\mathrm{G}+\mathrm{NX}$

Permite proyectar el crecimiento de la empresa respecto al desarrollo económico del país. Este índice lo componen todos los sectores de la economía de un país, por tanto, determina el mínimo crecimiento que se puede exigir a la empresa.

Los conjuntos difusos que se consideran para medir el PIB son:

PIB alto, economías que se caracterizan por tener una mejor calidad de vida y utilizan plenamente el potencial y los recursos. 
PIB medio, las personas tiene una calidad de vida promedio.

PIB bajo, no se explotan eficientemente los recursos y existe un subdesarrollo en la economía.

\subsubsection{IPC}

Se relaciona con la variación en los precios, lo cual afecta directamente los ingresos de la empresa. En los modelos de valoración se utiliza para proyectar el comportamiento de los bienes relacionados directa o indirectamente con la operación de la empresa.

Los conjuntos difusos que se consideran para medir el IPC son:

IPC alto, representa un patrón marcado por las preferencias de artículos costosos, un crecimiento del mercado acelerado dado por la dinámica en los precios del mercado y refleja la capacidad adquisitiva de las personas.

IPC medio, gastos diarios de los consumidores se ajustan a las necesidades básicas, y como estas no cambian, la variación en los precios no es muy significativa.

IPC bajo, se encuentra en economías subdesarrolladas, poco dinámicas.

\subsubsection{Costo de deuda}

Empresas que para obtener recursos financieros hacen uso de los aportes que hacen sus dueños (equity) o de aportes que hacen terceros (deuda), como proveedores, empleados, sistema financiero, etc.

El que invierte capital en la empresa lo hace esperando que le rente la inversión a una tasa que difiere para cada inversionista, en función del riesgo percibido en el negocio y del costo de oportunidad de cada uno [20].

Los conjuntos difusos que se consideran para medir el costo de deuda son:

Costo de deuda alto, costo superior al promedio y refleja una mala política de financiamiento.

Costo de deuda medio, se ajusta al valor del sector o de empresas similares.

Costo de deuda bajo, la empresa posee una fuerte capacidad de negociación.

La base de conocimiento para la medición los aspectos macroeconómicos debe presentar todas las combinaciones posibles, pero una parte se presenta en la tabla 5 . 
Tabla 5. Base de conocimientos para la variable competencias monetarias.

\begin{tabular}{|c|c|c|c|}
\hline \multicolumn{3}{|c|}{$I F$} & THEN \\
\hline IPC & Kd & PIB & Macroeconomicas \\
\hline Bajo & Bajo & Bajo & Bajas \\
\hline Bajo & Bajo & Medio & Altas \\
\hline Bajo & Bajo & Altas & Altas \\
\hline
\end{tabular}

Fuente: elaboración propia

\subsection{Variable de salida: inversión en la empresa}

La valoración de una empresa, desde el análisis de la inversión, podría dividirse en dos: el análisis cuantitativo y el cualitativo. Entrelazados permiten al inversionista tomar una decisión. La valoración integra cada uno de los componentes analizados: competencias internas, competencias monetarias y aspectos macroeconómicos.

El sistema de inferencia difuso para esta variable se presenta en la figura 5.

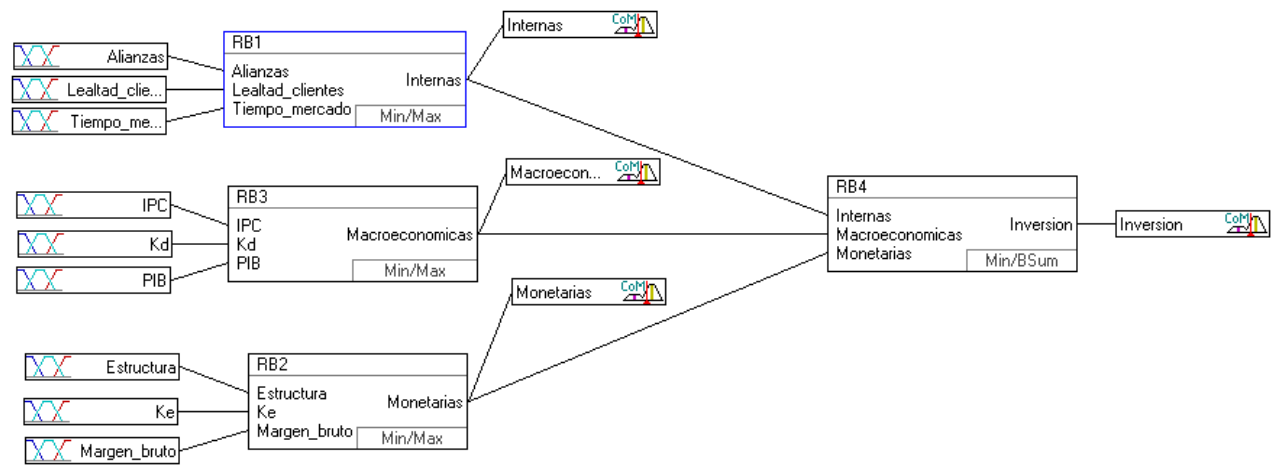

Figura 5. Estructura del sistema de inferencia para la inversión en la empresa.

Fuente: elaboración propia

Para la variable inversión en la empresa se consideran dos conjuntos difusos definidos como sigue:

Invertir, la empresa tiene competencias estratégicas que le ayudarán a lograr el cumplimiento de sus metas futuras, tanto en generación de beneficios como en crecimiento organizacional.

No invertir, incrementan en el analista la percepción de un entorno macroeconómico contraproducente para la empresa.

La base de conocimiento completa para la medición de los aspectos macroeconómicos se presenta en la tabla 6 . 
Tabla 6. Base de conocimientos para la variable inversión en la empresa.

\begin{tabular}{|c|c|c|c|}
\hline \multicolumn{3}{|c|}{ IF } & THEN \\
\hline Internas & Macroeconomicas & Monetarias & Inversion \\
\hline Bajas & Bajas & Bajas & No \\
\hline Bajas & Bajas & Altas & No \\
\hline Bajas & Altas & Bajas & No \\
\hline Bajas & Altas & Altas & Si \\
\hline Altas & Bajas & Bajas & No \\
\hline Altas & Bajas & Altas & Si \\
\hline Altas & Altas & Bajas & Si \\
\hline Altas & Altas & Altas & $\mathrm{Si}$ \\
\hline
\end{tabular}

Fuente: elaboración propia

\subsubsection{Superficies difusas}

Las superficies difusas que muestran las relaciones entre las competencias internas, las monetarias y los aspectos macroeconómicos, y su efecto sobre la decisión e inversión se muestran en la figura 6:

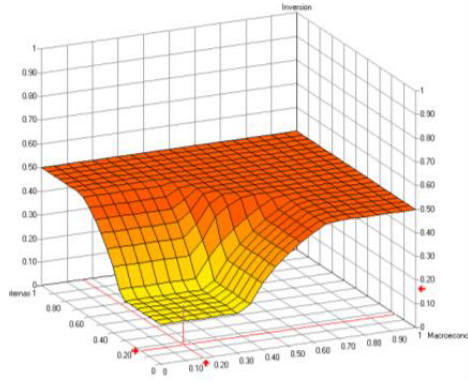

a) Internas vs. macroeconómicas

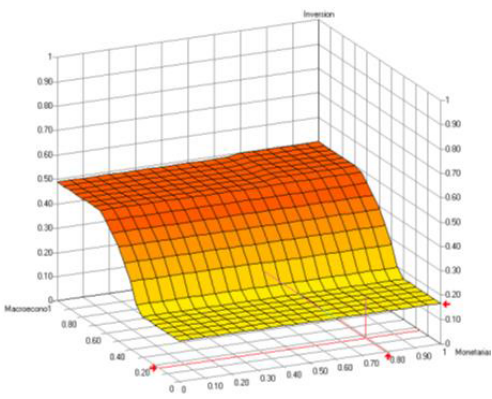

b) Monetarias y macroeconómica

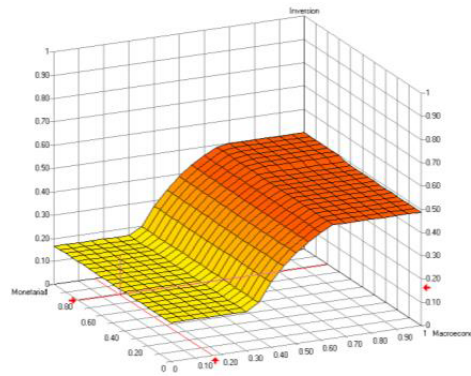

c) Monetarias e internas

Figura 6. Superficies difusas de las relaciones entre componentes de la valoración.

Fuente: elaboración propia 
De las superficies se aprecia que las competencias internas y macroeconómicas tienen un efecto constante sobre la decisión de inversión, interpretando como si el analista no fuera a cambiar la valoración por factores de mercado o propios de la empresa, pero sí tiene un impacto considerable: ingresar el aspecto financiero.

\section{CONCLUSIONES}

La herramienta presentada en esta investigación es un apoyo en la toma de decisiones de inversión, una herramienta de seguimiento a la gestión que permite medir las variaciones en el valor de la empresa originado por decisiones organizacionales.

Se recomienda el uso de esta herramienta como principal apoyo de la valoración, ya que los modelos determinísticos, como el flujo de caja descontado, no reflejan ni involucran todas las variables que impactan el valor de una empresa y que deberían ser considerados por el analista.

Esta herramienta es flexible y puede ajustarse a las características propias de un país o sector, ya que no tiene valores determinísticos que limiten su aplicación.

La herramienta integra variables que inicialmente estaban desagregadas e incorpora la percepción del analista, lo que resulta en una valoración genérica similar incluso entre diferentes analistas.

El modelo presenta el limitante del número de variables, ya que la elección de las variables definitivas debe ser el resultado de un minucioso análisis que considere las características propias de la empresa.

Futuros estudios del presente trabajo deberían centrarse en la inclusión de variables perceptivas de los clientes y en la determinación de variables originadas por otros modelos de valoración no mencionados en esta investigación.

El modelo permite trabajar con más de una variable o un dato, a diferencia de los modelos tradicionales que centran generalmente en el valor presente de los beneficios o en la generación de valor como principal respaldo a la decisión.

\section{REFERENCIAS}

[1] P. Viñolas, y X. Adserà. Principios de valoración de empresas. Bilbao: Ediciones Deusto, 2003, 314 pp.

[2] R. D. Alvares, et al., "Razones para valorar una empresa y los métodos empleados”, Semestre económico, vol. 9, $n .^{\circ} 18$, pp. 59-83, 2006.

[3] J. L. Martín, y A. Trujillo. Manual de valoración de empresas, 1. a ed., Barcelona: Editorial Ariel, 2000. 
[4] M. Alzate, y I. C. Hernández, Métodos para valoración de empresas que no cotizan en la bolsa de valores (Tesis para el grado Magíster en Administración). Universidad de Medellín, Medellín, 2006.

[5] P. Fernández. Valoración de empresas : cómo medir y gestionar la creación de valor. Barcelona: Gestión 2000, 2004.

[6] J.-S.R. Jang, C.-T. Sun y E. Mizutani. Neuro-fuzzy and soft computing: a computational approach to learning and machine intelligence. Upper Saddle River, NJ: Prentice Hall, 1997.

[7] A. D. Kulkarni. Computer vision and fuzzy, neural systems. Upper Saddle River, NJ: Prentice Hall PTR, 2001, 509 p.

[8] N. K. Kasabov. Foundations of neural networks, fuzzy systems, and knowledge engineering. Cambridge, Mass.: MIT Press, 1998.

[9] B. Kosko. Pensamiento borroso : la nueva ciencia de la lógica borrosa. Barcelona: Crítica. 1995.

[10] L. Wang. "Fuzzy systems are universal approximators". Presented at the IEEE Conference on Fuzzy Systems. San Diego, CA. Pp. 1163-1170, 1992.

[11] A. Kaufmanny y J. Gil. Las matemáticas del azar y de la incertidumbre : elementos básicos para su aplicación en economía. Madrid: Centro de Estudios Ramón Areces. 1990.

[12] E. Trillas. Conjuntos borrosos. Barcelona: Editorial Vicens-vives. 1980.

[13] H. R. Varian, y M. E. Rabasco. Microeconomía intermedia: un enfoque actual. Barcelona: Antoni Bosch. 1999.

[14] F. J. López, y W. De Luna. Valoración de empresas en la práctica. Madrid; Buenos Aires: McGraw-Hill Interamericana. 2001.

[15] M. A. Hitt et al. Administración estratégica : competitividad y globalización: conceptos y casos. México: Thomson, 2007.

[16] T. Bañegil, y F. Miranda, "Tiempo de mercado como determinante del éxito de nuevos productos: mito o realidad," Investigaciones Europeas de Dirección y Economía de la Empresa, vol. $7, n .^{\circ}$ 2, pp. 109-122, 2001.

[17] E. A. Helfert. Financial analysis : tools and techniques : a guide for managers. New York: McGraw-Hill, 2001.

[18] A. Damodaran. Damodaran on Valuation: Security Analysis for Investment and Corporate Finance. Hoboken, N.J: John Wiley \& Sons, 2006.

[19] S. Mongrut, Fuenzalida et al., "Explorando teorías de estructura de capital en Latinoamérica," Cuadernos de administración, vol. 23, n. ${ }^{\circ}$ 41, pp. 163-184. 2010.

[20] Z. Radi, y A. Bolívar. "Creación de valor de las empresas colombianas durante el período 2000-2005," Pensamiento y gestión. 22. 2007.

[21] L. J. Álvarez, y M. Sebastián, "La inflación permanente y latente en España: una perspectiva macroeconómica," Revista Española de Economía, vol. 15, n. ํ 1, pp. 37-65, 1998.

[22] V. Caballer, y E. Ballestero. Métodos de valoración de empresas, Madrid: Pirámide, 1998. 
[23] A. Medina. "Evaluación de la gestión del valor en una empresa: propuesta de un modelo," Theoria, vol. 11, pp. 43-50, 2002.

[24] E. Santandreu. Manual práctico de valoración de empresas. Barcelona: EADA. 1990. 